\title{
Parental smoking during pregnancy and offspring bone mass at age 10 years: findings from a prospective birth cohort
}

\author{
C. Macdonald-Wallis • J. H. Tobias • G. Davey Smith • \\ D. A. Lawlor
}

Received: 16 June 2010 / Accepted: 8 September 2010 / Published online: 22 October 2010

(C) The Author(s) 2010. This article is published with open access at Springerlink.com

\begin{abstract}
Summary We investigated an intrauterine influence of maternal smoking during pregnancy on childhood bone mass. Daughters, but not sons, of mothers who smoked had higher bone mass at age 10 years. This appears to be due to familial factors related to parental smoking influencing increased offspring adiposity rather than a direct intrauterine effect.

Introduction Neonatal studies have demonstrated an adverse relationship between maternal smoking in pregnancy and foetal bone mineral accrual. We aimed to investigate an intrauterine influence of maternal smoking during pregnancy on offspring bone mass at mean age 9.9 years.

Methods We compared associations of maternal and paternal smoking in pregnancy with offspring total body less head (TBLH) and spine bone mineral content (BMC), bone area (BA), bone mineral density (BMD) and area-adjusted BMC (ABMC) in 7,121 children in the Avon Longitudinal Study of Parents and Children.

Results Maternal smoking in any trimester was associated with increased TBLH BMC, BA and BMD in girls (mean difference $[95 \% \mathrm{CI}]$ (sex-specific SD scores), $0.13[0.05-$
\end{abstract}

Electronic supplementary material The online version of this article (doi:10.1007/s00198-010-1415-y) contains supplementary material, which is available to authorized users.

C. Macdonald-Wallis $(\bowtie) \cdot G$. Davey Smith $\cdot$ D. A. Lawlor MRC Centre for Causal Analyses in Translational Epidemiology, Department of Social Medicine, University of Bristol,

Oakfield House, Oakfield Grove,

Bristol BS8 2BN, UK

e-mail: C.Macdonald-Wallis@bristol.ac.uk

J. H. Tobias

Academic Rheumatology, Clinical Science at North Bristol, University of Bristol,

Bristol, UK
$0.22], 0.13$ [0.04-0.21], 0.13 [0.04-0.22], respectively) but not boys $(0.01$ [-0.07-0.09], 0.00 [-0.08-0.08], 0.04 $[-0.05-0.12])$, and also with spine $\mathrm{BMC}, \mathrm{BA}$ and $\mathrm{BMD}$ in girls $(0.13$ [0.03-0.23], 0.12 [0.03-0.22], 0.10 [0.00$0.21])$ but not boys $(0.03$ [-0.06-0.12], 0.00 [-0.09-0.09], $0.05[-0.04-0.14])$, but not with ABMC. Paternal smoking associations were similar, with no statistical evidence for a difference between maternal and paternal effects. Maternal associations increased on adjustment for offspring birth weight and gestational age, but attenuated to the null after adjustment for current height and weight.

Conclusions We found little evidence that maternal smoking was related to bone mass in boys. In girls, maternal smoking associations were similar to those of paternal smoking, suggesting that these were attributable to shared familial characteristics, not intrauterine mechanisms.

Keywords ALSPAC - Bone mineral content (BMC) . Bone mineral density (BMD) - Childhood · Pregnancy . Smoking

\section{Introduction}

The development of bone mass throughout childhood is important in determining the peak bone mass achieved in early adulthood [1], and simulation models have demonstrated the potential of small increases in peak bone mass to delay the onset of osteoporosis and therefore decrease the risk of fracture in the elderly [2]. Even during childhood, a reduction in bone mass is associated with an increased fracture risk from all levels of trauma severity [3, 4].

Maternal smoking during pregnancy has been shown to have a detrimental influence on the accrual of bone mass in utero. Two studies in the Southampton Women's Survey 
reported associations between maternal smoking and decreased whole body bone mineral content (BMC) in neonatal offspring $[5,6]$. The earlier of the two studies also found a similar relationship with bone mineral density (BMD) [5], but the more recent and larger study did not [6]. Little is known about longer term effects, although in a Tasmanian cohort of 330 participants, relationships were found between maternal smoking during pregnancy and reduced offspring femoral neck and lumbar spine BMC and BMD at age 8 years which remained after adjustment for current weight and height [7].

We assessed the associations of maternal smoking in pregnancy with the skeletal size and bone density at mean age 9.9 years of a large cohort of children: the Avon Longitudinal Study of Parents and Children (ALSPAC). We compared the effects of maternal smoking with those of paternal smoking during pregnancy since the paternal exposure would not be expected to influence foetal development via an intrauterine mechanism. Hence, stronger maternal associations would provide evidence of a direct intrauterine effect on bone development, whilst similar-sized maternal and paternal associations would indicate relationships driven by shared familial, social, genetic and environmental factors. This method has been used effectively to study the influences of maternal smoking on other outcomes in the ALSPAC [8-10], and its validity is demonstrated by the much greater association of maternal compared with paternal smoking in pregnancy with offspring birth weight, which is known to be influenced by maternal smoking via an intrauterine mechanism [11].

\section{Materials and methods}

\section{The ALSPAC}

The ALSPAC is a prospective birth cohort study aiming to investigate environmental and inheritable influences on the health and development of children. It has been previously described in full elsewhere and on the web site www.alspac. bris.ac.uk. Pregnant women with expected delivery dates between 1 April 1991 and 31 December 1992 and living in a defined area of Avon including the city of Bristol were eligible for recruitment to the study. A total of 14,541 women were enrolled, and 13,678 of these had a singleton live birth. Ethical approval for the study was obtained from the ALSPAC Law and Ethics Committee and from local ethics committees. At age 9 years, all children with known addresses who were still participating were invited to a "Focus@9" clinic, and 7,121 of the singleton children attended. Of these, 6,868 underwent a full-body dualenergy X-ray absorptiometry (DXA) scan.

\section{DXA measurements}

Whole body DXA scans were carried out using a Lunar Prodigy scanner (GE Healthcare Bio-Sciences Corp., USA), and after exclusion of those containing artefacts, movement or skeletal irregularities, there remained 6,775 scans. The total body less head (TBLH) region was used to represent the child's total bone mass, with the head excluded as bone development here is different from the rest of the skeleton and less likely to be influenced by environmental factors. In contrast to the overall skeleton which primarily comprises cortical bone, the spine subregion which was also analysed has a relatively high proportion of trabecular bone. One operator reanalysed the scans to check and adjust automated placement of body regions; in the case of the spinal region, the upper border comprises the cervicothoracic junction, the lower border the lumbosacral junction, and the lateral borders the bone/soft tissue interface. Since curvature in the image of the spine leads to contamination of the spinal region with the ribs, only images with minor or no curvature are included in the analysis of spinal outcomes. Measurements for TBLH and spine BMC, bone area (BA) and areal BMD were subsequently calculated. For both regions, area-adjusted BMC (ABMC) was also derived as a measure of volumetric BMD by using linear regression to adjust BMC for BA and adding the residuals to the mean $\mathrm{BMC}$ for the region. The coefficient of variation for TBLH BMD was $0.84 \%$ based on 122 pairs of scans repeated on the same day. At the same time as the DXA scan, the child's standing height (without shoes) was measured using a Harpenden Stadiometer (Holtain Ltd., UK) and weight (unshod and in light clothing) was measured using a Tanita Body Fat Analyzer (model TBF 305, Tanita UK Limited, UK).

\section{Maternal and paternal smoking}

At 18 weeks' gestation, the mothers were sent a postal questionnaire which asked how many times per day they had smoked in the first trimester and in the last 2 weeks, representing smoking during the second trimester. At 32 weeks' gestation, another postal questionnaire asked how many cigarettes per day the woman was currently smoking, representing smoking during the third trimester. Variables describing smoking in any trimester and smoking in all trimesters were derived from these responses, with one or more cigarettes smoked per day considered as smoking regularly. A questionnaire completed by the mother's partner at 18 weeks' gestation asked if he had smoked regularly at any time in the last 9 months. The mother was also asked if her partner smoked in her 18week questionnaire, and a positive response from either the partner or the mother was assumed sufficient to indicate that the partner smoked regularly during the pregnancy. 
Other variables

Maternal and paternal height, weight and highest educational qualifications, household social class, father's age and the mother's parity were obtained from questionnaires administered during pregnancy. Household social class was defined from the highest parental occupation, on a scale from I to $\mathrm{V}$, with I indicating a professional/ managerial role and $\mathrm{V}$ being unskilled manual. Maternal and paternal body mass index (BMI) were calculated as weight $(\mathrm{kg}) /$ height $(\mathrm{m})^{2}$. The child's sex was obtained at the time of birth, and the child's birth weight, gestational age and the mother's age at delivery were abstracted from obstetric records.

In the questionnaire administered at 18 weeks' gestation, the mother was asked how many hours per week she spent engaging in strenuous physical activity. The questionnaire also asked the number of hours per week the mother spent in a number of specific types of leisure activity, each of which was assigned a MET score [12], and a weighted activity index was developed by multiplying the MET score by the number of hours of activity per week. Dietary information for the mothers was obtained from a food frequency questionnaire administered at 32 weeks' gestation which asked how often they consumed each of the 43 food groups. Using nutrient information on standard-sized portions, the mother's total weekly energy, carbohydrate, fat and protein intakes were derived [13]. Although the main analysis did not adjust for these variables, since the equivalent paternal information was not available, an additional analysis was performed in which the relationships of maternal smoking in pregnancy with offspring bone outcomes were adjusted for maternal physical activity (strenuous activity of $3 \mathrm{~h}$ or more per week and weighted activity index) and diet (weekly energy, carbohydrate, fat and protein intake) during pregnancy.

Pubertal stage data for the children were obtained from Tanner stage questionnaires administered to the parents at 116 months and were based on pubic hair development for boys and breast development for girls, or pubic hair development if this was unavailable. For girls, age at menarche was derived from a series of questionnaires administered between the ages of 8 and 17 years which asked if the daughter had started her menstrual periods and, if so, the age she was at her first menstrual period. Where there was disagreement between questionnaires, the age given on the earliest questionnaire was used. Most children (99\% of boys and $96 \%$ of girls) with pubertal stage information were either pre- or early pubertal (Tanner stage 1 or 2). For this reason, and due to the high proportion of missing pubertal stage data, this has not been adjusted for in the main regression analysis, but an additional analysis was performed which adjusted for pubertal stage and, for girls, whether menarche occurred at age $\leq 10$ years.

\section{Paternity}

If, when asked in a questionnaire administered in pregnancy, the mother had not confirmed her partner to be the child's biological father, all paternal information (smoking status, BMI, age, height and education) was treated as missing.

\section{Statistical analysis}

We assessed maternal and paternal smoking associations with offspring bone outcomes separately and also in combined mutually adjusted regression models. We used sex-specific models due to evidence of interactions of maternal and paternal smoking with the child's sex and adjusted first for the child's age only, then additionally for the potential confounders of household social class, parity and maternal/paternal age, height, BMI and education. Maternal factors were included in maternal exposure models, paternal factors in paternal exposure models, and both maternal and paternal factors in combined models. To explore mediating relationships, we additionally adjusted for the child's birth weight and gestational age and then finally included the child's height and weight as potential mediators. Since there was little change in regression coefficients between the simple age-adjusted model and the model adjusting for all potential confounding factors (full results for all four models available from authors), only the confounder-adjusted model (age and all other potential confounders, model 1) and the two additional models exploring potential mediation by birth weight and gestational age (model 2) and by weight and height at age 9.9 (model 3) are presented. Sex-specific standard deviation (SD) scores of TBLH and spine BMC, BA, BMD and ABMC were used as outcomes.

We used multivariate multiple imputation of missing data to impute data for all children who attended the 9year clinic and also analysed the complete cases with no missing data on any of the exposures, outcomes or covariates to compare findings from the fully observed data with those from partially imputed data. Multiple imputation was used to increase the efficiency of the model estimates and reduce selection bias, which can be present in complete case analysis when data are not missing completely at random. The multiple imputation method is valid provided that the reasons for missingness in the data can be explained by other observed variables [14]. Detailed methods for this procedure are described in the Electronic supplementary material (ESM). All analyses were carried out in Stata version 11.0 (StataCorp LP, USA). 


\section{Results}

Table 1 shows the characteristics of the 7,121 children who attended the 9-year clinic. There were 6,101 sets of parents for whom both maternal and paternal smoking information was available; for $3,576(58.6 \%)$ of these neither parent smoked, for $369(6.0 \%)$ only the mother smoked, for 1,313 $(21.5 \%)$ only the father smoked, and for $843(13.8 \%)$ both parents smoked. Mothers who smoked at any time during pregnancy were younger and shorter on average, more likely to be of a manual social class and less likely to have an A-level or higher qualification than mothers who did not smoke (ESM Web Table 2). Pre-pregnancy BMI did not differ between mothers who smoked and those who did not. Children of mothers who smoked were lighter at birth and older, heavier and had higher fat mass at the time of the DXA scan on average.

Pairwise correlations of total body and spinal bone measures are given in ESM Web Table 3, and correlations of these measures with child and parental characteristics are shown in ESM Web Table 4. The child's height and weight were strongly positively correlated with TBLH and spine BMC and BA and moderately with TBLH and spine BMD. Higher birth weight, longer gestation and greater age at DXA scan were all associated with increased TBLH BMC, BA and BMD.

Multiple imputation analysis of maternal and paternal smoking in relation to TBLH bone outcomes is shown in Table 2 and the analysis of spinal outcomes shown in Table 3. In girls, maternal smoking in any trimester was associated with increased TBLH and spine BMC, BA and $\mathrm{BMD}$ in confounder-adjusted models (model 1). Mean differences in TBLH BMC and BA were slightly higher for mothers who smoked in all trimesters of pregnancy, but other associations were similar. In boys, maternal smoking in any trimester was not robustly associated with any TBLH or spinal bone outcomes. $P$ values for sex differences were $0.007,0.003$ and 0.085 for TBLH BMC, BA and $\mathrm{BMD}$ and $0.036,0.035$ and 0.119 for spine BMC, BA and $\mathrm{BMD}$, respectively, in models with maternal smoking in any trimester as the exposure. In combined confounderadjusted models (model 1) for girls, there were greater paternal smoking associations with TBLH BMC, BA and $\mathrm{BMD}$ and spine BMD compared with those for maternal smoking, whilst maternal associations were larger than paternal associations with spine BMC and BA. However, all $P$ values for differences between maternal and paternal effects were $>0.15$. There were no strong associations of paternal smoking with bone outcomes in boys. On additional adjustment for the child's birth weight and gestational age (model 2), there were increases in maternal associations, whilst paternal associations did not change. In boys, maternal smoking in all trimesters was positively associated with TBLH BMC, BA and BMD after adjustment for birth weight and gestational age. In fully adjusted models including offspring height and weight at age 9.9 years (model 3), all maternal relationships attenuated to the null, although a weak association remained with spine BA in girls. Paternal associations were similarly attenuated, and although evidence remained of an association with TBLH BA, this weakened in combined models. There were no associations between parental smoking during pregnancy and TBLH or spine ABMC, except for a weak positive association between paternal smoking and spine ABMC in girls. These models are not included in the tables (full data available from authors on request). Variance inflation factors for maternal smoking and paternal smoking in mutually adjusted models were all $<2$, indicating that there was low colinearity between the two parental smoking variables in these models.

To examine whether the associations in girls were mediated mainly by the child's height or weight, we adjusted for each individually (plus confounders). On adjustment for height without weight, mean differences in TBLH BMC, BA and BMD associated with maternal smoking in any trimester were $0.13 \mathrm{SD}, 0.12 \mathrm{SD}$ and 0.12 SD, respectively (all $P<0.01$ ). However, on adjustment for weight without height, mean differences were -0.02 $\mathrm{SD},-0.03 \mathrm{SD}$ and $0.00 \mathrm{SD}$ (all $P>0.2$ ), suggesting that the positive associations of maternal smoking with offspring bone mass are driven by the child's weight at age 9.9 years. Mean differences in TBLH BMC, BA and BMD associated with paternal smoking on adjustment for height without weight were $0.10 \mathrm{SD}, 0.10 \mathrm{SD}$ and $0.10 \mathrm{SD}$ (all $P<$ 0.01 ), and adjusting for weight without height were 0.01 $\mathrm{SD}, 0.01 \mathrm{SD}$ and $0.03 \mathrm{SD}$, respectively (all $P>0.2$ ). A similar pattern occurred in spine BMC, BA and BMD.

In complete case analysis (ESM Web Tables 5 and 6), associations of maternal smoking with TBLH and spinal $\mathrm{BMC}, \mathrm{BA}$ and $\mathrm{BMD}$ were equivalent to those using multiple imputation, but associations of paternal smoking were generally smaller in girls (by up to $0.07 \mathrm{SD}$ ). No strong associations of maternal or paternal smoking in pregnancy with bone outcomes were found in boys in the complete case in confounder-adjusted models. In combined confounder-adjusted models for TBLH bone outcomes in girls in the complete case maternal and paternal smoking associations were of a similar size, with little evidence for a difference between parental effects, as in multiple imputation models. However, in models for spinal bone outcomes, there were greater maternal compared with paternal associations, and there was statistical evidence for a difference between parental smoking associations with spinal BA. ESM Web Tables 7 and 8 compare the characteristics of multiply imputed and complete case datasets for TBLH and spinal bone outcomes, respectively, 
Table 1 Characteristics of children who attended the clinic at age 9 years and their parents $(N=7,121)$

\begin{tabular}{|c|c|c|c|c|}
\hline \multicolumn{3}{|l|}{ Characteristic } & $N$ with data $(\%)$ & Mean (SD) or median (IQR) or\% \\
\hline \multicolumn{5}{|l|}{ Child } \\
\hline Age at DXA scan (months) & & & $6,851(96.2)$ & $118.4(3.9)$ \\
\hline \multirow[t]{2}{*}{$\operatorname{Sex}(\%)$} & \multicolumn{2}{|l|}{ Male } & $7,121(100.0)$ & 49.6 \\
\hline & \multicolumn{2}{|l|}{ Female } & & 50.4 \\
\hline \multicolumn{3}{|l|}{ Height (cm) } & $7,047(99.0)$ & $139.5(6.3)$ \\
\hline \multicolumn{3}{|l|}{ Weight (kg) } & $7,105(99.8)$ & $33.2(29.4-38.4)^{\mathrm{a}}$ \\
\hline \multicolumn{3}{|l|}{ TBLH BMC (g) } & $6,775(95.1)$ & $893.8(184.0)$ \\
\hline \multicolumn{3}{|l|}{ TBLH BA $\left(\mathrm{cm}^{2}\right)$} & $6,775(95.1)$ & $1139.5(164.3)$ \\
\hline \multicolumn{3}{|l|}{ TBLH BMD $\left(\mathrm{g} / \mathrm{cm}^{2}\right)$} & $6,775(95.1)$ & $0.78(0.05)$ \\
\hline \multicolumn{3}{|l|}{ TBLH ABMC } & $6,775(95.1)$ & $894.6(39.8)$ \\
\hline \multicolumn{3}{|l|}{ Spine BMC (g) } & $5,487(77.1)$ & $78.4(15.7)$ \\
\hline \multicolumn{3}{|l|}{ Spine BA $\left(\mathrm{cm}^{2}\right)$} & $5,487(77.1)$ & $100.7(12.0)$ \\
\hline \multicolumn{3}{|l|}{ Spine BMD $\left(\mathrm{g} / \mathrm{cm}^{2}\right)$} & $5,487(77.1)$ & $0.77(0.08)$ \\
\hline \multicolumn{3}{|l|}{ Spine ABMC (g) } & $5,487(77.1)$ & $78.4(7.1)$ \\
\hline \multirow[t]{6}{*}{ Pubertal stage (\%) } & Boys & Tanner 1 & $2,365(67.0)$ & 82.9 \\
\hline & & Tanner 2 & & 16.5 \\
\hline & & Tanner 3+ & & 0.6 \\
\hline & Girls & Tanner 1 & $2,836(79.0)$ & 81.5 \\
\hline & & Tanner 2 & & 15.0 \\
\hline & & Tanner $3+$ & & 3.5 \\
\hline \multirow[t]{2}{*}{ Age at menarche for girls (years) $(\%)$} & Up to 10 & & $3,107(86.5)$ & 4.7 \\
\hline & $11+$ & & & 95.3 \\
\hline \multicolumn{3}{|l|}{ Gestational age (weeks) } & $7,121(100.0)$ & $39.5(1.8)$ \\
\hline \multicolumn{3}{|l|}{ Birth weight $(\mathrm{kg})$} & $7,035(98.8)$ & $3.4(0.5)$ \\
\hline \multirow[t]{5}{*}{ Household social class $(\%)$} & $\mathrm{I}$ & & $6,544(91.9)$ & 15.5 \\
\hline & II & & & 45.1 \\
\hline & III NM & & & 24.8 \\
\hline & III M & & & 10.3 \\
\hline & $\mathrm{IV} / \mathrm{V}$ & & & 4.3 \\
\hline \multicolumn{5}{|l|}{ Mother } \\
\hline \multicolumn{3}{|l|}{ Age at delivery (years) } & $7121(100.0)$ & $29.0(4.6)$ \\
\hline Height $(\mathrm{cm})$ & & & $6753(94.8)$ & $164.1(6.6)$ \\
\hline Pre-pregnancy BMI (kg/m²) & & & $6429(90.3)$ & $22.2(20.5-24.4)^{\mathrm{a}}$ \\
\hline No. of previous births (\%) & 0 & & $6879(96.6)$ & 45.8 \\
\hline & 1 & & & 35.5 \\
\hline & 2 & & & 13.7 \\
\hline & 3 & & & 3.8 \\
\hline & 4 or more & & & 1.2 \\
\hline Smoking during pregnancy (\%) & Never & & $6379(89.6)$ & 78.7 \\
\hline & 1 or 2 trimesters & & & 9.5 \\
\hline & All trimesters & & & 11.8 \\
\hline Education (\%) & None/CSE & & $6860(96.3)$ & 13.8 \\
\hline & Vocational & & & 8.5 \\
\hline & O Levels & & & 35.2 \\
\hline & A Levels & & & 26.6 \\
\hline & Degree & & & 15.8 \\
\hline Father & & & & \\
\hline Age at child's birth (years) & & & $5106(71.7)$ & $31.4(5.2)$ \\
\hline Height $(\mathrm{cm})$ & & & $4931(69.2)$ & $176.3(6.9)$ \\
\hline
\end{tabular}


Table 1 (continued)

\begin{tabular}{llcc}
\hline Characteristic & & $N$ with data (\%) & Mean (SD) or median (IQR) or\% \\
\hline BMI (kg/m $\left.{ }^{2}\right)$ & & $4887(68.6)$ & $24.8(22.9-26.9)^{\mathrm{a}}$ \\
Regular smoker (\%) & No & $6679(93.8)$ & 65.3 \\
& Yes & & 34.7 \\
Education (\%) & None/CSE & $6467(90.8)$ & 19.3 \\
& Vocational & & 8.2 \\
& O Levels & & 21.7 \\
& A Levels & & 28.5 \\
\hline
\end{tabular}

$A B M C$ area-adjusted bone mineral content, $B A$ bone area, $B M C$ bone mineral content, $B M D$ bone mineral density, $B M I$ body mass index, $I Q R$ interquartile range, $T B L H$ total body less head

${ }^{a}$ Median and interquartile range are shown for skewed variables

and show that parental educational qualifications tended to be higher in the complete case. We thus investigated the relationships between maternal and paternal smoking and TBLH and spinal BMC, BA and BMD in girls in the complete case and stratified the analysis into two subgroups: families where neither parent had an A-level or higher qualification and families where one or both parents was qualified to A level or above (data not shown). In TBLH models, paternal associations were greater than maternal associations in the stratum with lower parental qualifications, whilst maternal associations were greater in the stratum with higher parental qualifications. In the stratum with less educated parents, there were similarsized parental smoking associations with spinal bone outcomes, but greater maternal associations in the higher educated stratum. This suggests that the dissimilarities between the multiple imputation and complete case analyses are likely to be due to the differing parental education distributions in the datasets.

Of the 6,741 children whose ethnicity was known, $6,470(96.0 \%)$ were white. Restricting the analysis to children of known white ethnicity did not meaningfully change the model coefficients. Including maternal diet and physical activity during pregnancy in the multiple imputation process and additionally adjusting for these variables in models with maternal smoking as the exposure did not alter the findings. When we repeated the multiple imputation process with pubertal stage (for both boys and girls) and age of menarche (for girls only) included and additionally adjusted for these variables, model coefficients were similar for boys. In models with maternal smoking as the exposure for girls, associations were attenuated by up to $0.07 \mathrm{SD}$ compared with the original multiple imputation analysis, whilst associations of paternal smoking were unchanged.

\section{Discussion}

We compared the relationships of maternal and paternal smoking during pregnancy with offspring bone mass at mean age 9.9 years in a large birth cohort and found similar-sized associations of smoking in both parents with increased total body and spinal BMC, BA and areal BMD in girls, but little evidence for any associations in boys.

Maternal smoking during pregnancy was associated with $0.10-0.13$ SD increases in TBLH and spinal BMC, BA and BMD in daughters. These relationships were masked by the negative association of maternal smoking with the child's birth weight and gestational age and increased on adjustment for these factors, whilst effect sizes associated with paternal smoking did not change. This may be due to the negative intrauterine effect on the accrual of bone mass by the foetus [5, 6], which is unique to the maternal smoking exposure. Maternal smoking during pregnancy is known to lead to a smaller child at birth, both through an increased risk of preterm birth and through intrauterine growth retardation $[15,16]$, and a positive relationship has been reported between birth weight and BMD at the femoral neck and lumbar spine in 8-year-old children [17].

Conversely, relationships of maternal and paternal smoking with offspring bone mass attenuated to the null when the child's height and weight were included in regression models. BMC, BA and BMD are all related to bone size (as BMD is incompletely adjusted for bone area) and therefore correlate strongly with height and weight. Since no relationships were found between maternal smoking and ABMC, which reflects 'volumetric' BMC, it appears that the associations are working through skeletal size rather than density. The relationships were driven mainly by offspring weight, concurring with studies which have demonstrated an association between maternal smok- 
Table 2 Sex-specific associations of maternal and paternal smoking with total body less head bone outcomes at age 9.9 years in multiple imputation analysis (boys $N=3,530$; girls $N=3,591$ )

\begin{tabular}{|c|c|c|c|c|c|c|c|c|c|c|}
\hline & & Mean difference & $95 \% \mathrm{CI}$ & $P$ value & Mean difference & $95 \% \mathrm{CI}$ & $P$ value & Mean difference & $95 \% \mathrm{CI}$ & $P$ value \\
\hline \multicolumn{2}{|l|}{ Boys } & \multicolumn{3}{|c|}{ TBLH BMC (SD score: $1 \mathrm{SD}=174.6 \mathrm{~g}$ ) } & \multicolumn{3}{|c|}{ TBLH BA (SD score: $1 \mathrm{SD}=154.9 \mathrm{~cm}^{2}$ ) } & \multicolumn{3}{|c|}{ TBLH BMD (SD score: $1 \mathrm{SD}=0.053 \mathrm{~g} / \mathrm{cm}^{2}$ ) } \\
\hline \multicolumn{11}{|c|}{ Maternal smoking in any trimester } \\
\hline \multicolumn{2}{|l|}{ Model 1} & 0.01 & $-0.07-0.09$ & 0.767 & 0.00 & $-0.08-0.08$ & 0.992 & 0.04 & $-0.05-0.12$ & 0.419 \\
\hline \multicolumn{2}{|l|}{ Model 2} & 0.05 & $-0.03-0.14$ & 0.186 & 0.05 & $-0.03-0.13$ & 0.232 & 0.06 & $-0.03-0.15$ & 0.177 \\
\hline \multicolumn{2}{|l|}{ Model 3} & 0.00 & $-0.05-0.04$ & 0.885 & -0.01 & $-0.04-0.03$ & 0.736 & 0.01 & $-0.06-0.08$ & 0.752 \\
\hline \multicolumn{11}{|c|}{ Maternal smoking in all trimesters } \\
\hline \multicolumn{2}{|c|}{ Model 1} & 0.07 & $-0.04-0.17$ & 0.200 & 0.05 & $-0.05-0.15$ & 0.356 & 0.10 & $-0.01-0.21$ & 0.086 \\
\hline \multicolumn{2}{|l|}{ Model 2} & 0.13 & $0.02-0.23$ & 0.016 & 0.12 & $0.01-0.22$ & 0.025 & 0.13 & $0.02-0.24$ & 0.020 \\
\hline \multicolumn{2}{|l|}{ Model 3} & 0.00 & $-0.06-0.05$ & 0.877 & -0.02 & $-0.06-0.03$ & 0.482 & 0.03 & $-0.06-0.12$ & 0.523 \\
\hline \multicolumn{11}{|c|}{ Paternal smoking } \\
\hline \multicolumn{2}{|c|}{ Model 1} & 0.02 & $-0.05-0.10$ & 0.519 & 0.03 & $-0.04-0.10$ & 0.405 & 0.01 & $-0.07-0.08$ & 0.887 \\
\hline \multicolumn{2}{|l|}{ Model 2} & 0.03 & $-0.04-0.10$ & 0.425 & 0.04 & $-0.03-0.11$ & 0.305 & 0.01 & $-0.07-0.08$ & 0.833 \\
\hline \multicolumn{2}{|l|}{ Model 3} & -0.02 & $-0.05-0.02$ & 0.357 & -0.01 & $-0.04-0.02$ & 0.581 & -0.03 & $-0.09-0.03$ & 0.313 \\
\hline \multicolumn{11}{|c|}{ Combined models } \\
\hline \multirow[t]{2}{*}{ Model 1} & Maternal smoking ${ }^{a}$ & 0.01 & $-0.08-0.09$ & 0.830 & -0.01 & $-0.09-0.08$ & 0.888 & 0.04 & $-0.05-0.13$ & 0.396 \\
\hline & Paternal smoking & 0.03 & $-0.04-0.10$ & 0.465 & 0.04 & $-0.03-0.11$ & 0.298 & 0.00 & $-0.08-0.08$ & 0.985 \\
\hline \multirow[t]{2}{*}{ Model 2} & Maternal smoking ${ }^{a}$ & 0.05 & $-0.04-0.13$ & 0.277 & 0.04 & $-0.04-0.12$ & 0.369 & 0.06 & $-0.03-0.16$ & 0.194 \\
\hline & Paternal smoking & 0.02 & $-0.05-0.09$ & 0.588 & 0.03 & $-0.04-0.10$ & 0.409 & -0.01 & $-0.08-0.07$ & 0.894 \\
\hline \multirow[t]{2}{*}{ Model 3} & Maternal smoking ${ }^{a}$ & 0.00 & $-0.04-0.05$ & 0.925 & 0.00 & $-0.04-0.03$ & 0.845 & 0.02 & $-0.05-0.10$ & 0.523 \\
\hline & Paternal smoking & -0.02 & $-0.06-0.02$ & 0.383 & -0.01 & $-0.04-0.02$ & 0.644 & -0.03 & $-0.10-0.03$ & 0.266 \\
\hline \multicolumn{2}{|l|}{ Girls } & \multicolumn{3}{|c|}{ TBLH BMC (SD score: $1 \mathrm{SD}=191.5 \mathrm{~g}$ ) } & \multicolumn{3}{|c|}{ TBLH BA (SD score: $1 \mathrm{SD}=172.3 \mathrm{~cm}^{2}$ ) } & TBLH BMD (SD s & ore: $1 \mathrm{SD}=0.0$ & $\left.55 \mathrm{~g} / \mathrm{cm}^{2}\right)$ \\
\hline Maternal s & moking in any trime & ster & & & & & & & & \\
\hline Model 1 & & 0.13 & $0.05-0.22$ & 0.003 & 0.13 & $0.04-0.21$ & 0.004 & 0.13 & $0.04-0.22$ & 0.005 \\
\hline Model 2 & & 0.17 & $0.08-0.25$ & $<0.001$ & 0.17 & $0.08-0.25$ & $<0.001$ & 0.15 & $0.06-0.24$ & 0.001 \\
\hline Model 3 & & 0.02 & $-0.02-0.06$ & 0.384 & 0.02 & $-0.01-0.06$ & 0.205 & 0.02 & $-0.04-0.08$ & 0.528 \\
\hline Maternal s & moking in all trimes & & & & & & & & & \\
\hline Model 1 & & 0.15 & $0.03-0.26$ & 0.011 & 0.15 & $0.04-0.26$ & 0.009 & 0.13 & $0.01-0.24$ & 0.037 \\
\hline Model 2 & & 0.20 & $0.09-0.32$ & 0.001 & 0.21 & $0.10-0.32$ & $<0.001$ & 0.16 & $0.04-0.28$ & 0.008 \\
\hline Model 3 & & 0.02 & $-0.03-0.07$ & 0.371 & 0.03 & $-0.01-0.08$ & 0.127 & 0.01 & $-0.07-0.09$ & 0.871 \\
\hline Paternal sr & noking & & & & & & & & & \\
\hline Model 1 & & 0.15 & $0.08-0.22$ & $<0.001$ & 0.14 & $0.08-0.21$ & $<0.001$ & 0.14 & $0.07-0.21$ & $<0.001$ \\
\hline Model 2 & & 0.16 & $0.09-0.23$ & $<0.001$ & 0.15 & $0.09-0.22$ & $<0.001$ & 0.15 & $0.07-0.22$ & $<0.001$ \\
\hline Model 3 & & 0.03 & $-0.00-0.07$ & 0.058 & 0.03 & $0.00-0.06$ & 0.029 & 0.04 & $-0.02-0.09$ & 0.164 \\
\hline Combined & models & & & & & & & & & \\
\hline Model 1 & Maternal smoking ${ }^{a}$ & 0.10 & $0.01-0.19$ & 0.025 & 0.10 & $0.01-0.19$ & 0.030 & 0.10 & $0.01-0.19$ & 0.032 \\
\hline & Paternal smoking & 0.12 & $0.05-0.20$ & 0.001 & 0.12 & $0.05-0.19$ & 0.002 & 0.12 & $0.04-0.19$ & 0.004 \\
\hline Model 2 & Maternal smoking ${ }^{a}$ & 0.13 & $0.04-0.22$ & 0.004 & 0.13 & $0.04-0.22$ & 0.003 & 0.12 & $0.03-0.21$ & 0.011 \\
\hline & Paternal smoking & 0.12 & $0.05-0.19$ & 0.001 & 0.12 & $0.05-0.19$ & 0.001 & 0.11 & $0.04-0.19$ & 0.004 \\
\hline Model 3 & Maternal smoking ${ }^{a}$ & 0.01 & $-0.03-0.05$ & 0.670 & 0.01 & $-0.02-0.05$ & 0.457 & 0.01 & $-0.05-0.08$ & 0.706 \\
\hline & Paternal smoking & 0.03 & $-0.01-0.06$ & 0.101 & 0.03 & $-0.00-0.06$ & 0.087 & 0.04 & $-0.02-0.10$ & 0.198 \\
\hline
\end{tabular}

Model 1 is adjusted for the child's age, mother's parity, household social class and maternal/paternal factors (age, height, pre-pregnancy BMI, education).

Model 2 is adjusted additionally for the child's gestational age and birth weight

Model 3 is adjusted for all these plus the child's height and weight at age 9.9 years

Reference category for maternal smoking variables is "Never smoked during pregnancy" and for paternal smoking variable is "Non-smoking" $B A$ bone area, $B M C$ bone mineral content, $B M D$ bone mineral density, $T B L H$ total body less head

${ }^{a}$ Maternal smoking in any trimester 
Table 3 Sex-specific associations of maternal and paternal smoking with spinal bone outcomes at age 9.9 years in multiple imputation analysis (boys $N=2,772$; girls $N=2,715$ )

\begin{tabular}{|c|c|c|c|c|c|c|c|c|c|c|}
\hline & & Mean difference & $95 \% \mathrm{CI}$ & $P$ value & Mean difference & $95 \% \mathrm{CI}$ & $P$ value & Mean difference & $95 \% \mathrm{CI}$ & $P$ value \\
\hline \multicolumn{2}{|l|}{ Boys } & \multicolumn{3}{|c|}{ Spine BMC (SD score: $1 \mathrm{SD}=14.8 \mathrm{~g}$ ) } & \multicolumn{3}{|c|}{ Spine BA (SD score: $1 \mathrm{SD}=11.7 \mathrm{~cm}^{2}$ ) } & \multicolumn{3}{|c|}{ Spine BMD (SD score: $\left.1 \mathrm{SD}=0.076 \mathrm{~g} / \mathrm{cm}^{2}\right)$} \\
\hline \multicolumn{11}{|c|}{ Maternal smoking in any trimester } \\
\hline \multicolumn{2}{|c|}{ Model 1} & 0.03 & $-0.06-0.12$ & 0.501 & 0.00 & $-0.09-0.09$ & 0.918 & 0.05 & $-0.04-0.14$ & 0.304 \\
\hline \multicolumn{2}{|l|}{ Model 2} & 0.07 & $-0.02-0.16$ & 0.153 & 0.05 & $-0.04-0.14$ & 0.289 & 0.07 & $-0.03-0.16$ & 0.171 \\
\hline \multicolumn{2}{|l|}{ Model 3} & 0.01 & $-0.05-0.07$ & 0.683 & 0.01 & $-0.04-0.07$ & 0.640 & 0.01 & $-0.07-0.09$ & 0.829 \\
\hline \multicolumn{11}{|c|}{ Maternal smoking in all trimesters } \\
\hline \multicolumn{2}{|c|}{ Model 1} & 0.06 & $-0.06-0.18$ & 0.301 & 0.04 & $-0.08-0.15$ & 0.534 & 0.07 & $-0.05-0.20$ & 0.245 \\
\hline \multicolumn{2}{|l|}{ Model 2} & 0.11 & $-0.01-0.23$ & 0.063 & 0.10 & $-0.02-0.21$ & 0.100 & 0.10 & $-0.03-0.22$ & 0.122 \\
\hline \multicolumn{2}{|l|}{ Model 3} & -0.02 & $-0.09-0.06$ & 0.640 & -0.01 & $-0.09-0.06$ & 0.745 & -0.01 & $-0.12-0.09$ & 0.792 \\
\hline \multicolumn{11}{|c|}{ Paternal smoking } \\
\hline \multicolumn{2}{|c|}{ Model 1} & 0.03 & $-0.06-0.11$ & 0.535 & 0.03 & $-0.05-0.11$ & 0.404 & 0.00 & $-0.08-0.09$ & 0.950 \\
\hline \multicolumn{2}{|l|}{ Model 2} & 0.03 & $-0.05-0.11$ & 0.421 & 0.04 & $-0.04-0.12$ & 0.283 & 0.01 & $-0.08-0.09$ & 0.884 \\
\hline \multicolumn{2}{|l|}{ Model 3} & -0.01 & $-0.06-0.04$ & 0.634 & 0.01 & $-0.04-0.05$ & 0.834 & -0.03 & $-0.10-0.04$ & 0.346 \\
\hline \multicolumn{11}{|c|}{ Combined models } \\
\hline \multirow[t]{2}{*}{ Model 1} & Maternal smoking ${ }^{\mathrm{a}}$ & 0.05 & $-0.05-0.14$ & 0.344 & 0.01 & $-0.08-0.11$ & 0.802 & 0.07 & $-0.03-0.17$ & 0.166 \\
\hline & Paternal smoking & 0.02 & $-0.07-0.10$ & 0.706 & 0.03 & $-0.05-0.11$ & 0.458 & -0.01 & $-0.10-0.08$ & 0.797 \\
\hline \multirow[t]{2}{*}{ Model 2} & Maternal smoking ${ }^{\mathrm{a}}$ & 0.07 & $-0.02-0.17$ & 0.127 & 0.05 & $-0.05-0.14$ & 0.311 & 0.08 & $-0.02-0.19$ & 0.106 \\
\hline & Paternal smoking & 0.01 & $-0.07-0.09$ & 0.774 & 0.03 & $-0.05-0.11$ & 0.526 & -0.01 & $-0.10-0.08$ & 0.767 \\
\hline \multirow[t]{2}{*}{ Model 3} & Maternal smoking ${ }^{\mathrm{a}}$ & 0.02 & $-0.04-0.08$ & 0.537 & 0.02 & $-0.05-0.08$ & 0.642 & 0.03 & $-0.06-0.11$ & 0.557 \\
\hline & Paternal smoking & -0.02 & $-0.07-0.04$ & 0.548 & 0.00 & $-0.05-0.05$ & 0.997 & -0.04 & $-0.11-0.04$ & 0.330 \\
\hline \multicolumn{2}{|l|}{ Girls } & \multicolumn{3}{|c|}{ Spine BMC (SD score: $1 \mathrm{SD}=16.7 \mathrm{~g}$ ) } & \multicolumn{3}{|c|}{ Spine BA (SD score: $1 \mathrm{SD}=12.3 \mathrm{~cm}^{2}$ ) } & Spine BMD (SD sco & re: $1 \mathrm{SD}=0.08$ & $\left.36 \mathrm{~g} / \mathrm{cm}^{2}\right)$ \\
\hline Maternal s & moking in any trimes & & & & & & & & & \\
\hline Model 1 & & 0.13 & $0.03-0.23$ & 0.013 & 0.12 & $0.03-0.22$ & 0.012 & 0.10 & $0.00-0.21$ & 0.049 \\
\hline Model 2 & & 0.15 & $0.05-0.25$ & 0.002 & 0.16 & $0.06-0.25$ & 0.001 & 0.12 & $0.01-0.22$ & 0.025 \\
\hline Model 3 & & 0.02 & $-0.03-0.07$ & 0.444 & 0.05 & $-0.00-0.10$ & 0.065 & -0.01 & $-0.08-0.06$ & 0.799 \\
\hline Maternal s & moking in all trimeste & & & & & & & & & \\
\hline Model 1 & & 0.13 & $0.01-0.25$ & 0.035 & 0.12 & $-0.00-0.23$ & 0.055 & 0.11 & $-0.01-0.24$ & 0.081 \\
\hline Model 2 & & 0.18 & $0.06-0.30$ & 0.004 & 0.17 & $0.06-0.29$ & 0.004 & 0.14 & $0.01-0.26$ & 0.035 \\
\hline Model 3 & & 0.04 & $-0.02-0.11$ & 0.210 & 0.07 & $-0.00-0.13$ & 0.054 & 0.01 & $-0.09-0.10$ & 0.859 \\
\hline Paternal sr & noking & & & & & & & & & \\
\hline Model 1 & & 0.10 & $0.02-0.18$ & 0.014 & 0.08 & $-0.01-0.16$ & 0.066 & 0.12 & $0.04-0.20$ & 0.005 \\
\hline Model 2 & & 0.11 & $0.03-0.19$ & 0.009 & 0.08 & $0.00-0.16$ & 0.043 & 0.12 & $0.04-0.20$ & 0.004 \\
\hline Model 3 & & 0.01 & $-0.03-0.06$ & 0.580 & 0.00 & $-0.04-0.05$ & 0.951 & 0.03 & $-0.03-0.09$ & 0.288 \\
\hline Combined & models & & & & & & & & & \\
\hline Model 1 & Maternal smoking ${ }^{\mathrm{a}}$ & 0.11 & $0.01-0.21$ & 0.040 & 0.11 & $0.02-0.21$ & 0.020 & 0.07 & $-0.04-0.18$ & 0.186 \\
\hline & Paternal smoking & 0.07 & $-0.01-0.16$ & 0.089 & 0.05 & $-0.04-0.13$ & 0.293 & 0.10 & $0.01-0.18$ & 0.025 \\
\hline Model 2 & Maternal smoking ${ }^{\mathrm{a}}$ & 0.13 & $0.03-0.23$ & 0.013 & 0.14 & $0.05-0.24$ & 0.003 & 0.08 & $-0.02-0.19$ & 0.130 \\
\hline & Paternal smoking & 0.07 & $-0.01-0.15$ & 0.101 & 0.04 & $-0.04-0.13$ & 0.337 & 0.10 & $0.01-0.18$ & 0.027 \\
\hline Model 3 & Maternal smoking ${ }^{\mathrm{a}}$ & 0.02 & $-0.04-0.07$ & 0.545 & 0.06 & $-0.00-0.11$ & 0.058 & -0.02 & $-0.10-0.06$ & 0.546 \\
\hline & Paternal smoking & 0.01 & $-0.04-0.06$ & 0.681 & -0.01 & $-0.06-0.03$ & 0.598 & 0.04 & $-0.02-0.11$ & 0.197 \\
\hline
\end{tabular}

Reference category for maternal smoking variables is "Never smoked during pregnancy" and for paternal smoking variable is "Non-smoking" Model 1 is adjusted for the child's age, mother's parity, household social class and maternal/paternal factors (age, height, pre-pregnancy BMI, education).

Model 2 is adjusted additionally for the child's gestational age and birth weight

Model 3 is adjusted for all these plus the child's height and weight at age 9.9 years

$B A$ bone area, $B M C$ bone mineral content, $B M D$ bone mineral density

${ }^{a}$ Maternal smoking in any trimester 
ing in pregnancy and increased BMI and risk of overweight in childhood [15, 18-25], whilst the child's height deficit at birth has been shown to track to age 8 years [22]. Studies comparing the associations of maternal and paternal smoking in pregnancy with offspring BMI also report similar effects for both parental exposures [9, 24]. Furthermore, a previous study in ALSPAC found an inverse relationship of parental social position with offspring BMC and BA at age 9.9 years, also acting via the pathway of offspring weight [26]. It therefore seems most plausible that our associations are not explained by intrauterine effects, but rather that unmeasured aspects of the shared family environment which are associated with parental smoking, such as diet or level of physical activity, influence increased weight gain and greater bone mass in the children. Studies have shown that overweight children and adolescents have higher whole body and spinal bone mass [27-29] and that $\mathrm{BMC}$ is positively related to both lean and fat mass in childhood [30, 31]. Fat mass has been demonstrated to stimulate bone growth in prepubertal children previously in the ALSPAC $[32,33]$.

There has been a greater association reported between fat mass and bone mineral accrual in girls than in boys during puberty [34, 35], which may in part explain why we found no associations in boys, although one study suggests that this sex difference is not present in prepubertal children [35]. In our cohort, there was also a weaker univariate relationship between maternal smoking and offspring weight in sons than in daughters, so it is also possible that the social characteristics in families where parents smoke have a lesser influence on adiposity in boys than girls. In analysis adjusted for pubertal stage (both genders) and age at menarche (in girls), the associations between maternal smoking and bone outcomes in girls were attenuated, whereas the paternal associations remained similar. This suggests that these positive maternal associations may partly be explained by the association between maternal smoking in pregnancy and earlier age at menarche, which has been shown previously in ALSPAC [36]. Adjustment for pubertal stage in boys did not affect the associations between parental smoking and bone outcomes, and parental smoking was not related to pubertal stage at age 10 years in boys.

Our findings conflict with the study by Jones et al. [7] which indicated negative relationships between maternal smoking in pregnancy and bone mass in 8-year-olds for the total body, femoral neck and lumbar spine, with relationships at the femoral neck and lumbar spine remaining after adjustment for the child's height and weight. However, they studied a Tasmanian cohort identified at birth as at increased risk of sudden infant death syndrome which contained $65 \%$ male offspring and a higher prevalence of maternal smoking during pregnancy (49\%) compared with ours $(21 \%)$. Children of mothers who smoked were lighter at age 8 years in Jones' study, whereas we found a strong positive relationship between maternal smoking and offspring weight. Jones et al. do not make comparison with paternal smoking or give sex-specific findings.

The differing associations of parental smoking with spinal bone outcomes which we found in the complete case and multiple imputation analyses are probably due to the different populations represented by the two datasets since the complete case includes only around half of the children who attended the 9-year clinic. The rationale for comparing maternal and paternal smoking associations with offspring bone mass was that there is likely to be residual confounding in these relationships from unmeasured factors. Differing distributions of unmeasured confounders in the complete case and multiply imputed datasets could explain the difference between associations seen. Since there were differing educational distributions between the complete case and multiply imputed datasets and we found that parental smoking associations in the complete case differed between strata of parental education levels despite adjusting for all observed confounders, it seems that residual confounding is a possible explanation. Another possible reason for the difference is violation of the multiple imputation assumption that the missing data mechanisms can be explained by other observed variables. However, we verified that missingness in each of the variables with missing data was strongly associated with other observed variables and included a number of predictors of missingness in prediction equations to impute missing data. We therefore expect the multiply imputed datasets to be more representative of the study population and analyses based on these data more accurate.

A limitation to our study was the self-report of smoking by the mothers and fathers. Maternal smoking could be affected by reporting bias since mothers may be aware of the harmful effects of smoking and less likely to respond affirmatively. Nevertheless, where both the mother and father provided information about the father's smoking status, there was agreement in $94.5 \%$ of couples. The study benefitted from its large size, the ability to control for a number of potential confounders and the ability to compare associations of bone outcomes with both maternal and paternal exposures to assess the level of residual confounding.

\section{Conclusions}

Our study has found positive associations of maternal smoking during pregnancy with offspring total body and spinal bone mass in girls, with minimal evidence for any associations in boys, and our multivariable analyses and parental comparisons suggest that these associations are 
largely driven by familial characteristics related to childhood adiposity and unlikely to be due to intrauterine mechanisms. Although our findings do not demonstrate negative effects of maternal smoking in pregnancy on offspring bone mass, its known adverse effects for mothers and offspring health mean than women should be encouraged not to smoke.

Acknowledgments We are extremely grateful to all of the families who took part in this study, the midwives for their help in recruiting them and the whole ALSPAC team, which includes interviewers, computer and laboratory technicians, clerical workers, research scientists, volunteers, managers, receptionists and nurses.

\section{Conflicts of interest None.}

Funding The work presented in this paper was funded by Wellcome Trust grant number WT087997MA. Core support for ALSPAC is provided by the United Kingdom Medical Research Council, the Wellcome Trust and the University of Bristol. The UK Medical Research Council provides funding for the MRC Centre for Causal Analyses in Translational Epidemiology (G0600705).

Open Access This article is distributed under the terms of the Creative Commons Attribution Noncommercial License which permits any noncommercial use, distribution, and reproduction in any medium, provided the original author(s) and source are credited.

\section{References}

1. Cooper C, Cawley M, Bhalla A, Egger P, Ring F, Morton L, Barker D (1995) Childhood growth, physical-activity, and peak bone mass in women. J Bone Miner Res 10:940-947

2. Hernandez CJ, Beaupré GS, Carter DR (2003) A theoretical analysis of the relative influences of peak BMD, age-related bone loss and menopause on the development of osteoporosis. Osteoporos Int 14:843-847

3. Clark EM, Ness AR, Bishop NJ, Tobias JH (2006) Association between bone mass and fractures in children: a prospective cohort study. J Bone Miner Res 21:1489-1495

4. Clark EM, Ness AR, Tobias JH (2008) Bone fragility contributes to the risk of fracture in children, even after moderate and severe trauma. J Bone Miner Res 23:173-179

5. Godfrey K, Walker-Bone K, Robinson S, Taylor P, Shore S, Wheeler T, Cooper C (2001) Neonatal bone mass: influence of parental birthweight, maternal smoking, body composition, and activity during pregnancy. J Bone Miner Res 16:1694-1703

6. Harvey NC, Javaid MK, Arden NK, Poole JR, Crozier SR, Robinson SM, Inskip HM, Godfrey KM, Dennison EM, Cooper C, SWS Study Team (2010) Maternal predictors of neonatal bone size and geometry: the Southampton Women's Survey. J Dev Orig Health Dis 1:35-41

7. Jones G, Riley M, Dwyer T (1999) Maternal smoking during pregnancy, growth, and bone mass in prepubertal children. J Bone Miner Res 14:146-151

8. Leary S, Davey Smith G, Ness A (2006) Smoking during pregnancy and components of stature in offspring. Am J Hum Biol 18:502-512
9. Leary SD, Davey Smith G, Rogers IS, Reilly JJ, Wells JC, Ness AR (2006) Smoking during pregnancy and offspring fat and lean mass in childhood. Obesity (Silver Spring) 14:2284-2293

10. Brion MJA, Leary SD, Davey Smith G, Ness AR (2007) Similar associations of parental prenatal smoking suggest child blood pressure is not influenced by intrauterine effects. Hypertension 49:1422-1428

11. Davey Smith G (2008) Assessing intrauterine influences on offspring health outcomes: can epidemiological studies yield robust findings? Basic Clin Pharmacol Toxicol 102:245-256

12. Ainsworth BE, Haskell WL, Whitt MC, Irwin ML, Swartz AM, Strath SJ, O'Brien WL, Bassett DR, Schmitz KH, Emplaincourt PO, Jacobs DR, Leon AS (2000) Compendium of physical activities: an update of activity codes and MET intensities. Med Sci Sports Exerc 32:S498-S516

13. Rogers I, Emmett P (1998) Diet during pregnancy in a population of pregnant women in South West England. Eur J Clin Nutr 52:246-250

14. Rubin DB (1996) Multiple imputation after $18+$ years. J Am Stat Assoc 91:473-489

15. Vik T, Jacobsen G, Vatten L, Bakketeig LS (1996) Pre- and postnatal growth in children of women who smoked in pregnancy. Early Hum Dev 45:245-255

16. Floyd RL, Rimer BK, Giovino GA, Mullen PD, Sullivan SE (1993) A review of smoking in pregnancy - effects on pregnancy outcomes and cessation efforts. Annu Rev Public Health 14:379411

17. Jones G, Dwyer T (2000) Birth weight, birth length, and bone density in prepubertal children: evidence for an association that may be mediated by genetic factors. Calcif Tissue Int 67:304-308

18. Williams S, Poulton R (1999) Twins and maternal smoking: ordeals for the fetal origins hypothesis? A cohort study. Br Med J 318:897-900

19. Toschke AM, Koletzko B, Slikker W, Hermann M, von Kries R (2002) Childhood obesity is associated with maternal smoking in pregnancy. Eur J Pediatr 161:445-448

20. von Kries R, Toschke AM, Koletzko B, Slikker W (2002) Maternal smoking during pregnancy and childhood obesity. Am J Epidemiol 156:954-961

21. Wideroe M, Vik T, Jacobsen G, Bakketeig LS (2003) Does maternal smoking during pregnancy cause childhood overweight? Paediatr Perinat Epidemiol 17:171-179

22. Chen AM, Pennell ML, Klebanoff MA, Rogan WJ, Longnecker MP (2006) Maternal smoking during pregnancy in relation to child overweight: follow-up to age 8 years. Int $\mathrm{J}$ Epidemiol 35:121-130

23. Gilman SE, Gardener H, Buka SL (2008) Maternal smoking during pregnancy and children's cognitive and physical development: a causal risk factor? Am J Epidemiol 168:522-531

24. von Kries R, Bolte G, Baghi L, Toschke AM (2008) Parental smoking and childhood obesity - is maternal smoking in pregnancy the critical exposure? Int J Epidemiol 37:210-216

25. Nagel G, Wabitsch M, Galm C, Berg S, Brandstetter S, Fritz M, Klenk J, Peter R, Prokopchuk D, Steiner R, Stroth S, Wartha O, Weiland SK, Steinacker J (2009) Determinants of obesity in the Ulm Research on Metabolism, Exercise and Lifestyle in Children (URMEL-ICE). Eur J Pediatr 168:1259-1267

26. Clark EM, Ness A, Tobias JH (2005) Social position affects bone mass in childhood through opposing actions on height and weight. J Bone Miner Res 20:2082-2089

27. Goulding A, Taylor RW, Jones IE, Manning PJ, Williams SM (2002) Spinal overload: a concern for obese children and adolescents? Osteoporos Int 13:835-840

28. Rocher E, Chappard C, Jaffre C, Benhamou CL, Courteix D (2008) Bone mineral density in prepubertal obese and control 
children: relation to body weight, lean mass, and fat mass. J Bone Miner Metab 26:73-78

29. El Hage R, Jacob C, Moussa E, Benhamou CL, Jaffre C (2009) Total body, lumbar spine and hip bone mineral density in overweight adolescent girls: decreased or increased? J Bone Miner Metab 27:629-633

30. Ilich JZ, Skugor M, Hangartner T, An BS, Matkovic V (1998) Relation of nutrition, body composition and physical activity to skeletal development: a cross-sectional study in preadolescent females. J Am Coll Nutr 17:136-147

31. Goulding A, Taylor RW, Grant AM, Murdoch L, Williams SM, Taylor BJ (2008) Relationship of total body fat mass to bone area in New Zealand five-year-olds. Calcif Tissue Int 82:293-299

32. Clark EM, Ness AR, Tobias JH (2006) Adipose tissue stimulates bone growth in prepubertal children. J Clin Endocrinol Metab 91:2534-2541
33. Timpson NJ, Sayers A, Davey Smith G, Tobias JH (2009) How does body fat influence bone mass in childhood? A Mendelian randomization approach. J Bone Miner Res 24:522-533

34. Sayers A, Tobias JH (2010) Fat mass exerts a greater effect on cortical bone mass in girls than boys. J Clin Endocrinol Metab 95:699-706

35. Ackerman A, Thornton JC, Wang J, Pierson RN Jr, Horlick M (2006) Sex difference in the effect of puberty on the relationship between fat mass and bone mass in 926 healthy subjects, 618 years old. Obesity (Silver Spring) 14:819-825

36. Rubin C, Maisonet M, Kieszak S, Monteilh C, Holmes A, Flanders D, Heron J, Golding J, McGeehin M, Marcus M (2009) Timing of maturation and predictors of menarche in girls enrolled in a contemporary British cohort. Paediatr Perinat Epidemiol 23:492-504 